\title{
Delayed Cutaneous Hypersensitivity Reaction to Vaxzevria (ChAdOx1-S) Vaccine against SARS-CoV- 2
}

\section{Rosanne Sprute}

University of Cologne

\section{Sofie Schumacher}

University of Cologne

Martina Pauls

University of Cologne

Wolfgang Pauls

University of Cologne

Oliver A. Cornely ( $\boldsymbol{D}$ oliver.cornely@uk-koeln.de )

University of Cologne

\section{Short Report}

Keywords: AstraZeneca, Coronavirus vaccine, COVID-19, COVID arm, delayed-type reaction, Pfizer/BioNTechModerna, mRNA vaccine, T-cell mediated reaction, viral vector vaccine

Posted Date: May 11th, 2021

DOI: https://doi.org/10.21203/rs.3.rs-464753/v1

License: (c) (1) This work is licensed under a Creative Commons Attribution 4.0 International License.

Read Full License

Version of Record: A version of this preprint was published at Drugs in R\&D on August 5th, 2021. See the published version at https://doi.org/10.1007/s40268-021-00358-z. 


\section{Abstract}

Recently, an increasing number of cases with delayed large cutaneous reaction after immunisation with mRNA-based vaccines have been reported. This adverse reaction - which is considered a delayed-type or T-cell mediated hypersensitivity reaction - has been described primarily for the Moderna (mRNA-1273) vaccine and to a lesser extent for Comirnaty (Pfizer/BioNTech, BNT162b2).

We describe a delayed large cutaneous reaction in a patient who received the viral vector vaccine Vaxzevria (ChAdOx1-S). The time course and clinical symptoms of delayed major skin reaction after vaccination with Moderna or Pfizer/BioNTech have a similar pattern that we recognized in our patient after Vaxzevria vaccination. In contrast to the Moderna vaccine trials, this phenomenon has not been described in the Vaxzevria clinical trials and is to the best of our knowledge the first report of this adverse reaction to a vector-based vaccine against SARS-CoV-2.

With this, we hope to raise awareness about delayed injection site reactions that also occur after viral vector vaccines and to encourage additional reporting and patient education regarding the cutaneous reactions after COVID-19 vaccination.

\section{Main Text}

In January 2021, the European Medicines Agency (EMA) authorized use of the viral vector COVID-19 vaccine Vaxzevria (ChAdOx1-S) in the European Union. Given the scale-up of mass vaccination campaigns across the world, it is likely that new adverse reactions will occur that were not reported in the initial trials.

We have observed a delayed large local skin reaction to the first dose of Vaxzevria in a 62-year-old, white female. Except for cat allergy, no history of allergy was reported. Following the resolution of initial local and systemic symptoms after vaccination, the delayed reaction had an onset on day 10 that appeared near the injection site. The appearance of the reaction was an erythema with a maximum diameter of 10 $\mathrm{cm}$ on day 14 after vaccination (Figure 1). Considerable induration and pain near the injection site was reported. Besides the local erythema, no further cutaneous symptoms in other localizations were observed. The symptoms cleared up after 15 days and fully resolved over the following 3 days. No systemic symptoms occurred with delayed local reaction. The patient did not require treatment for reaction. A biopsy was not performed.

The complete post-vaccination course was as follows: On day 0 , the vaccination with Vaxzevria took place and no immediate reaction to the vaccine was observed. On day 1 after vaccination, the patient reported elevated temperature, malaise, and fatigue. The symptoms of systemic reaction resolved on day 2 , but a slight soreness near the injection site was reported. On day 3 , an erythema with $5 \mathrm{~cm}$ diameter, warmth and swelling was observed near injection site. On days 4-6, local symptoms remitted and completely resolved on day 7-9 except for a palpable nodule in the deep soft tissue. On day 10, a new erythema recurred near injection site and spread to a maximum diameter of $10 \mathrm{~cm}$ on days $11-14$, 
accompanied by a large, localized swelling and induration. From day 15 , the erythema was regressive and had completely resolved by day 18 . Photographs show the skin reaction over time with varied size and severity of the local findings between day 10 and 14 (Figure 1).

In the Vaxzevria clinical trials, a delayed local large reaction to the vaccine has not been described. In the phase $1 / 2$ trial of Vaxzevria, local site reactions were only recorded for 7 days following vaccination. ${ }^{1}$ It is therefore possible that the observation period was too short to report the delayed injection site reactions. Likewise, in the interim primary efficacy analysis of four phase 3 randomized controlled trials of ChAdOx1-S, no case with delayed cutaneous reaction was reported. ${ }^{2}$

On the other hand, a similar phenomenon as in our patient has been described multiple times for the mRNA-based Moderna (mRNA-1273) vaccine and to lesser extent for Comirnaty (Pfizer/BioNTech, BNT162b2). ${ }^{3-5}$ In the Moderna phase 3 trial, delayed injection site reaction occurred in $244(0 \cdot 8 \%)$ participants after the first dose of Moderna vaccine and in 68 (0 2\%) participants after the second dose. ${ }^{3}$ The delayed injection site reaction was defined as reaction on or after day 8 .

The International COVID-19 Dermatology Registry has started capturing skin reactions to COVID-19 vaccines in January 2021 and just recently published 414 cases with skin reactions to Comirnaty and Moderna vaccines. ${ }^{5}$ They defined delayed large local reactions as occurrence after 4 or more days after vaccination. The most common cutaneous reaction to Moderna vaccination was delayed large local reactions ( $n=175$ first dose, $n=31$ second dose). Interestingly, they also reported cases with local large reaction after administration of Comirnaty ( $n=5$ first dose, $n=7$ second dose). Of note, no cases of anaphylaxis or other serious adverse events after subsequent vaccination following delayed major local reactions to Moderna or Comirnaty vaccines were described. ${ }^{5}$

The time course and clinical symptoms of delayed major skin reaction after vaccination with Moderna or Comirnaty have a similar pattern that we recognized in our patient after Vaxzevria vaccination. To the best of our knowledge, this is the first description of a patient with delayed large local reaction to the vector-based Vaxzevria vaccine.

We suspect a delayed-type or T-cell-mediated hypersensitivity reaction as it was proposed for the mRNA vaccines. ${ }^{4}$ A skin-biopsy specimen obtained from a patient with delayed large local reaction after Moderna vaccination showed findings consistent with delayed-type or T-cell-mediated hypersensitivity, supporting this hypothesis. ${ }^{4}$ Although the aetiology of these reactions is unclear, delayed-type hypersensitivity reactions to excipient-ingredients of vaccines have been reported previously. ${ }^{5}$

Given that neither local injection site reactions nor delayed-type hypersensitivity reactions are contraindications to subsequent vaccination, our patient was encouraged to receive the second dose of Vaxzevria to complete her vaccination course. With this correspondence, we would like to raise awareness about delayed injection site reactions occurring also after viral vector vaccines against SARS- 
CoV-2 and hope to encourage additional reporting and patient education regarding the cutaneous reactions after COVID-19 vaccination.

\section{Declarations}

The patient gave her permission to use clinical information and photographs in the publication.

\section{Acknowledgement}

We gratefully acknowledge Prof. Manuel Cornely's support in describing the dermatologic findings of the delayed cutaneous reaction in our patient. We also thank Jan Thielebeule for technical support.

\section{Authors' contribution}

RS drafted the initial version of the manuscript, prepared the figure and reviewed and approved the final version of the manuscript. SS contributed to manuscript preparation and reviewed and approved the final version of the manuscript. MP provided the case description and photographs, reviewed and approved the final version of the manuscript. WP provided the case description and photographs, reviewed and approved the final version of the manuscript. OAC conceived the study idea, reviewed, and approved the final version of the manuscript.

\section{Declaration of interests}

RS, SS, MP and WP have nothing to disclose. OAC reports grants and personal fees from Actelion, personal fees from Allecra Therapeutics, personal fees from Al-Jazeera Pharmaceuticals, grants and personal fees from Amplyx, grants and personal fees from Astellas, grants and personal fees from Basilea, personal fees from Biosys, grants and personal fees from Cidara, grants and personal fees from Da Volterra, personal fees from Entasis, grants and personal fees from F2G, grants and personal fees from Gilead, personal fees from Grupo Biotoscana, personal fees from IQVIA, grants from Janssen, personal fees from Matinas, grants from Medicines Company, grants and personal fees from MedPace, grants from Melinta Therapeutics, personal fees from Menarini, grants and personal fees from Merck/MSD, personal fees from Mylan, personal fees from Nabriva, personal fees from Noxxon, personal fees from Octapharma, personal fees from Paratek, grants and personal fees from Pfizer, personal fees from PSI, personal fees from Roche Diagnostics, grants and personal fees from Scynexis, personal fees from Shionogi, grants from DFG, German Research Foundation, grants from German Federal Ministry of Research and Education, grants from Immunic, outside the submitted work.

\section{Source of funding}

The authors received no specific funding for this work.

\section{References}


1. Folegatti PM, Ewer KJ, Aley PK, Angus B, Becker S, Belij-Rammerstorfer S, et al. Safety and immunogenicity of the ChAdO $\times 1 \mathrm{nCoV}-19$ vaccine against SARS-CoV-2: a preliminary report of a phase 1/2, single-blind, randomised controlled trial. Lancet. 2020;396(10249):467-78.

2. Voysey M, Clemens SAC, Madhi SA, Weckx LY, Folegatti PM, Aley PK, et al. Safety and efficacy of the ChAdOx1 nCoV-19 vaccine (AZD1222) against SARS-CoV-2: an interim analysis of four randomised controlled trials in Brazil, South Africa, and the UK. Lancet. 2021;397(10269):99-111.

3. Baden LR, El Sahly HM, Essink B, Kotloff K, Frey S, Novak R, et al. Efficacy and Safety of the mRNA1273 SARS-CoV-2 Vaccine. N Engl J Med. 2021;384(5):403-16.

4. Blumenthal KG, Freeman EE, Saff RR, Robinson LB, Wolfson AR, Foreman RK, et al. Delayed Large Local Reactions to mRNA-1273 Vaccine against SARS-CoV-2. N Engl J Med. 2021;384(13):1273-7.

5. McMahon DE, Amerson E, Rosenbach M, Lipoff JB, Moustafa D, Tyagi A, et al. Cutaneous Reactions Reported after Moderna and Pfizer COVID-19 Vaccination: A Registry-Based Study of 414 Cases. J Am Acad Dermatol. 2021.

\section{Figures}

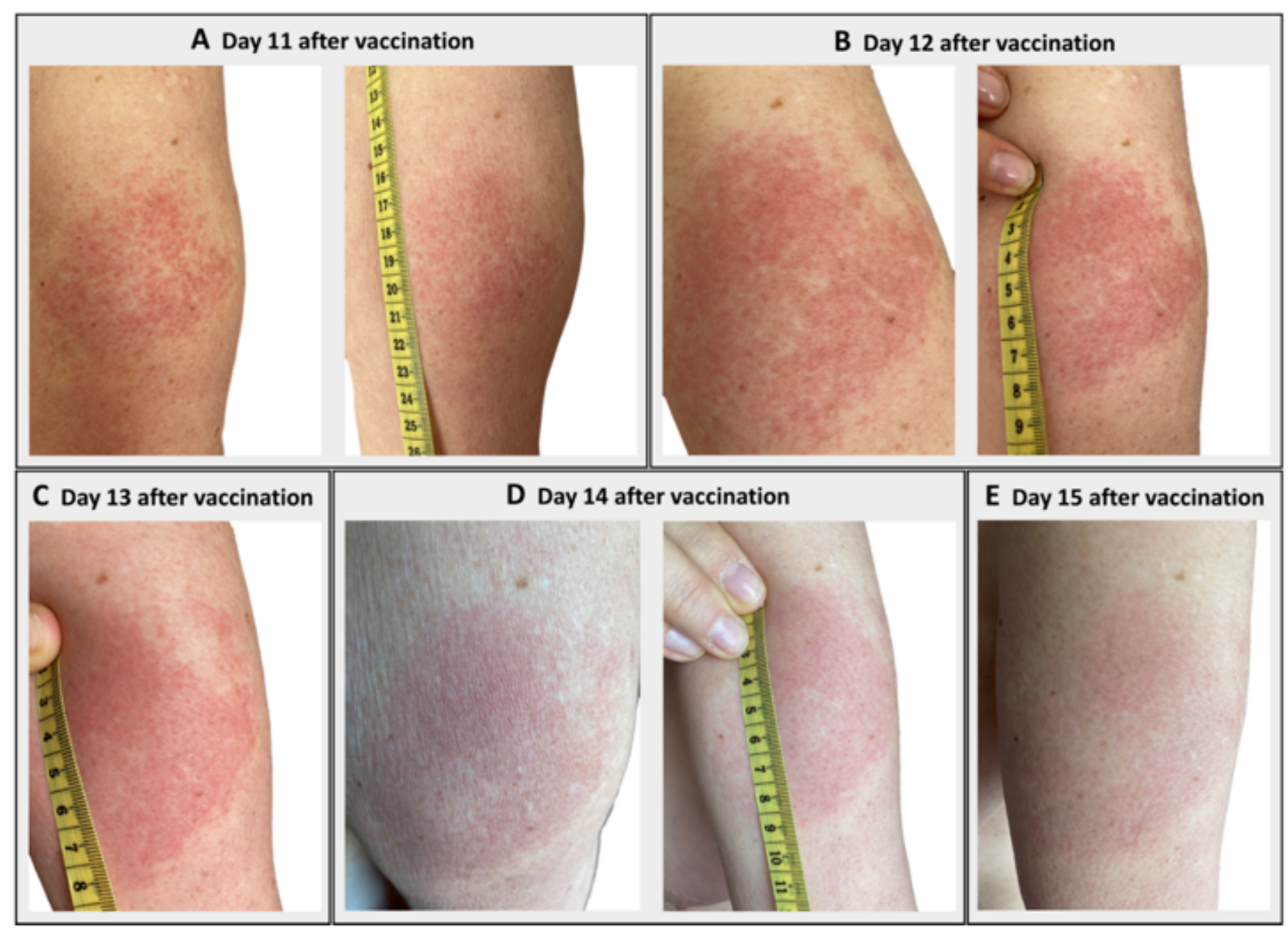




\section{Figure 1}

Photographs of the delayed large local cutaneous reaction that first occurred on day 10 after vaccination against SARS-CoV-2 with Vaxzevria. The cutaneous findings from (A) day 11 to (E) day 15 after vaccination are depicted. Morphologic characteristics included $(A)$ an erythematous macula with initially indistinct margin and follicular accentuated. The macula does not blanch with pressure. (B) The macula is extending centripetally and horizontally, at first without central fading, then (C) becoming confluent (DE) and with central fading in the course. Small skin wrinkles become visible again. The macula is surrounded by a pale peripheral margin. The lesion was up to $10 \mathrm{~cm}$ in diameter, with swelling and induration near the injection site of the vaccine. The symptoms resolved from day 15 onwards. 